\title{
The quest to reveal science's hidden female faces
}

Campaign on Wikipedia seeks to track down photos of female scientists and those from minority ethnic groups.

Dalmeet Singh Chawla

08 March 2017

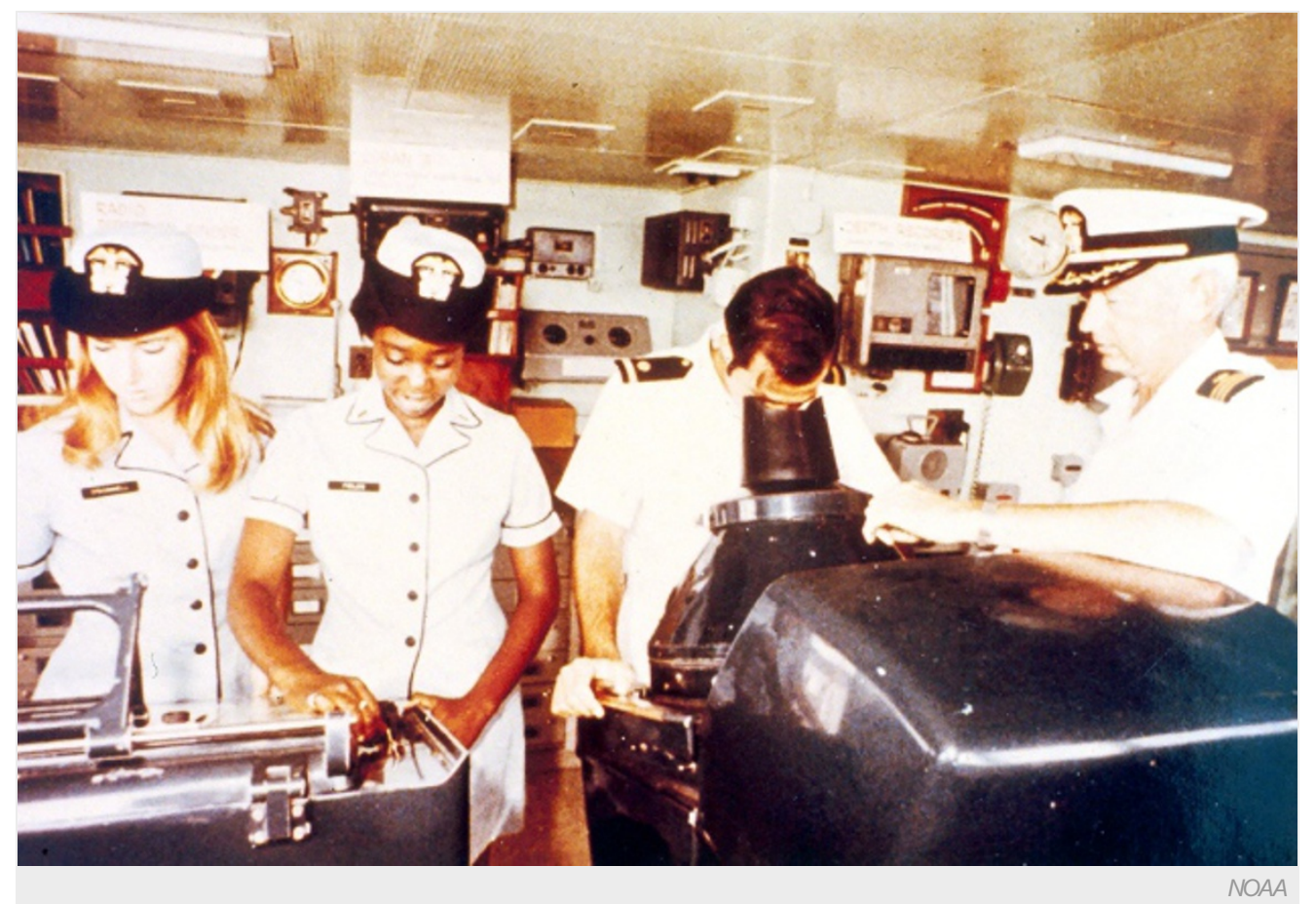

An effort to add photos of minority and female scientists to Wikipedia includes this photo of Evelyn Fields (second left, pictured in 1974). She is the first African-American and first woman to lead the Officer Corps at the US National Oceanic \& Atmospheric Administration.

Hilda Bastian was organizing a Wikipedia edit-a-thon for the US National Institutes of Health in 2015 to highlight the work of women scientists when she realized how difficult it was to find photos of them.

"I scanned through thousands of digitized photos in image galleries, went through Wikipedia pages, and looked for obituaries in journals. It had a big impact on me," says Bastian, who is based in Washington, DC and is an editor for PubMed Health, a health-information service from the US National Library of Medicine.

Bastian noticed a "stark bias" towards photos of men and white women scientists. "I could feel my own image of scientists in history changing. Who we see affects us," she says. 
So for Black History Month in February, she decided to track down copyright-free photos of African American women researchers and add them to their respective Wikipedia pages. Her project has continued into March — Women's History Month — and now includes scientists from other under-represented backgrounds. She also just launched an associated Twitter account, @MissingSciFaces.

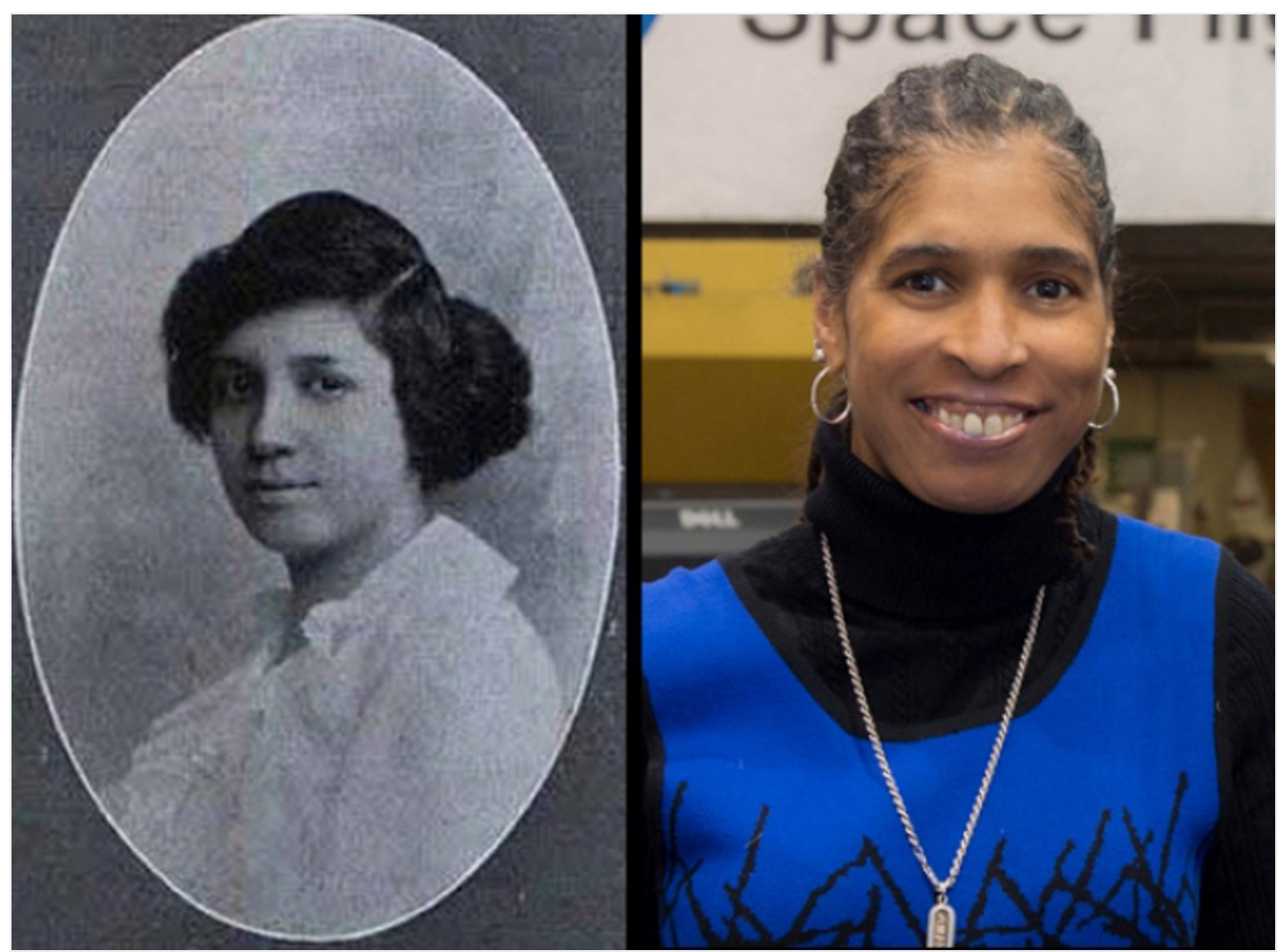

Ericsson: Bill Hybyk/KSFC/NASA, CC by 2.0

Euphemia Haynes (pictured in 1914), the first African-American woman to get a mathematics PhD; Aprille Ericsson-Jackson, a NASA aerospace engineer.

"It's so important for all of us to prevent invisibility in the historical and social record of scientists who always had the odds most stacked against them," Bastian writes in a blogpost on 6 March for PLOS, for which she is a contributor.

"It's remarkable how easy it is to overlook women," says Alice Dreger, a historian of science, who has tweeted approvingly about the project. "It is important to recognize the success of women in science, as well as the struggles they continue to face."

\section{Matilda effect}

Bastian wants others to help her, and has written a guide for sourcing images. A lot of material is available online, she writes, but it requires some digging. She recommends sifting through Google Images, Flickr Commons, Newspapers.com, or even approaching the researchers or their families or taking photos of female scientists at conferences.

She notes that getting copyright-free images is one problem facing the project: only public-domain images can go into a Wikipedia page, for example, via the site's repository Wikimedia Commons. 


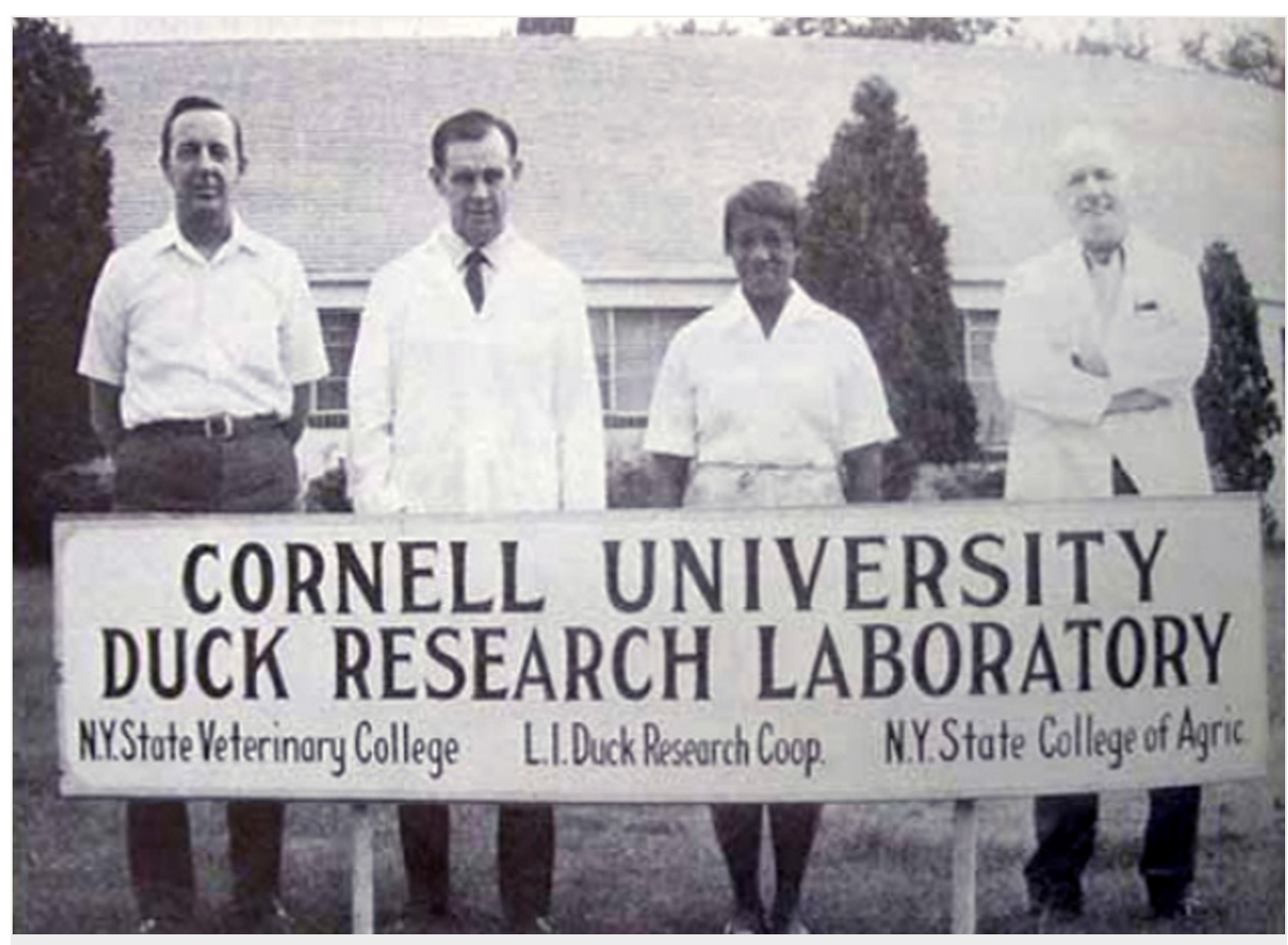

Intemational Duck Research Laboratory, Comell Univ.

Cornell University has released into the public domain this image of Jessie Price (second from right), a veterinary microbiologist.

Lenny Teytelman, cofounder and chief executive of the open-access scientific-methods repository Protocols.io, says that Bastian's project aims to address a phenomenon known as the 'Matilda Effect' - a bias against women in which their work is attributed to their male colleagues. "While the Matilda Effect is about the bias against women, I have no doubt that the bias against scientists from underrepresented groups is even more severe," he says. Bastian's effort is a small step, but it is "a very concrete and actionable how-to guide" to redressing the balance, he says.

This month, Bastian plans to systemize lists of African American women scientists on Wikipedia, and to encourage others to help identify people. Ultimately, she says, "It will be a success if it's no longer my personal project."

Nature I doi:10.1038/nature.2017.21614

Nature ISSN0028-0836 ESSN 1476-4687

\section{SPRINGER NATURE}

